\title{
A contact model for normal immersed collisions between a particle and a wall
}

\author{
Xiaobai Li, Melany L. Hunt $\dagger$ and Tim Colonius \\ Division of Engineering and Applied Science, California Institute of Technology, Pasadena, \\ CA 91125, USA
}

(Received 23 March 2011; revised 16 August 2011; accepted 16 October 2011; first published online 1 December 2011)

The incompressible Navier-Stokes equations are solved numerically to predict the coupled motion of a falling particle and the surrounding fluid as the particle impacts and rebounds from a planar wall. The method is validated by comparing the numerical simulations of a settling sphere with experimental measurements of the sphere trajectory and the accompanying flow field. The normal collision process is then studied for a range of impact Stokes numbers. A contact model of the liquid-solid interaction and elastic effect is developed that incorporates the elasticity of the solids to permit the rebound trajectory to be simulated accurately. The contact model is applied when the particle is sufficiently close to the wall that it becomes difficult to resolve the thin lubrication layer. The model is calibrated with new measurements of the particle trajectories and reproduces the observed coefficient of restitution over a range of impact Stokes numbers from 1 to 1000.

Key words: particle/fluid flows, suspensions

\section{Introduction}

Liquid-solid flows are involved in a wide variety of geophysical and industrial processes, including nearshore sediment transport, debris surges and landslides, the handling of dredging slurries and hydraulic fracture technologies (see Crowe, Sommerfeld \& Tsuji 1998; Lorenzini \& Mazza 2004). These flows are heterogeneous and the macroscopic behaviour of the mixture may not be described adequately by mixture theories that average over the fluid and solid phases. The rheology of liquid-solid mixtures depends on many factors, including the hydrodynamic forces, inter-particle interactions, the volume fraction, gravity, and the size and shape of particles. As an example, the erosion of bedrock results from impacts and abrasion by suspended sentiments; hence, a model of the process should include details at the particle level such as the kinetic energy of the impacting particles, the impact rates and angles, the material properties of the bedrock surface, as well as the properties of the suspending flow (see Lamb, Dietrich \& Sklar 2008).

Particle-particle and particle-wall collisions play an important role in a liquid-solid flow because they affect particle accumulation and dispersion, and inter-phase transport and mixing. Compared to a dry collision in which the fluid resistance is negligible and the particle inertia is dominant, the kinetic energy of a particle in a liquid environment 
is dissipated by viscous stresses in the liquid and by inelasticity during collision. The ratio of particle inertia to viscous forces is quantified through the Stokes number, $S t=(1 / 9)\left(\rho_{p} / \rho_{l}\right) R e$, where $R e=\rho_{l} D V / \mu$ is the particle Reynolds number based on impact velocity $V$, the particle diameter $D$, the liquid viscosity $\mu$, and the density of the particle and liquid $\rho_{p}$ and $\rho_{l}$ respectively.

By simultaneously accounting for elastic deformation and viscous forces, Davis, Serayssol \& Hinch (1986) established the range of conditions for deformation and rebound of colliding spheres. In their analysis, they demonstrated that the fluid pressure increases significantly as the particles approach, which results in a flattening of the particles and a decrease in particle velocity to zero. As the particles deform, some of kinetic energy of the incoming particle is converted into the elastic strain energy allowing the particles to rebound. As the particles move apart, fluid is drawn into the gap and the pressure drops, which hinders the motion of the rebounding spheres. Because the particles are perfectly smooth, they never contact each other during the collision process; the particles, however, reach a minimum distance of approach when they are separated by a thin lubrication layer. This minimum distance depends on the Stokes number and an elasticity parameter that quantifies the elastic properties of the colliding particles. The coefficient of restitution, $e$, defined as the ratio of the rebound to impact velocity, is strongly influenced by the Stokes number. For Stokes numbers less than a critical value, the particle does not rebound $(e=0)$ because of viscous dissipation of the initial kinetic energy. The critical Stokes number depends on the elasticity of the particles. For Stokes numbers greater than the critical value, $e$ is non-zero and increases to a value that also depends on the elasticity parameter. This work was extended by Barnocky \& Davis (1989) to include the effect of a fluid with pressure-dependent viscosity and density. They found that under extreme pressure building up in the thin fluid layer, the fluid may compress resulting in viscosity increasing by several orders of magnitude. These pressure-dependent effects may alter the minimum separation reached during approach of the spheres.

Barnocky \& Davis (1988) experimentally examined the impact of a sphere on a surface covered by a thin layer of liquid in order to investigate the critical Stokes number. The later work by Davis, Rager \& Good (2002) also used a thin layer of fluid but the investigators measured the impact and rebound velocity to determine the effective coefficient of restitution. In contrast, Joseph et al. (2001) measured the approach and rebound of a fully immersed collision between a particle suspended as a pendulum and a wall to determine the coefficient of restitution as a function of Stokes number. From their experimental measurements they showed that below a Stokes number of approximately 10, no rebound of the particle occurs. At a Stokes number greater than 1000, the particle rebound speed is not affected by the surrounding fluid. Hence, the coefficient of restitution, $e$, increases from 0 at $S t \approx 10$ to a dry value, $e_{d}$, which occurs for a collision with negligible fluid resistance. The dry coefficient of restitution depends on the properties of the solid phases and impact speed. The study by Joseph et al. (2001) measured $e_{d}$ of around 0.90 for glass, steel and Nylon particles colliding against a Lucite wall with impact speeds from 40 to $360 \mathrm{~mm} \mathrm{~s}^{-1}$; $e_{d}$ was higher (approximately 0.97) when these particles impacted a harder glass or Zerodur surface. The dependence of the coefficient of restitution on the Stokes number was also presented in the work by Gondret, Lance \& Petit (2002); in this study, the authors measured the normal trajectory of the particle over multiple bounces. Joseph \& Hunt (2004) examined oblique collisions between a particle and a wall. Later work by Yang $\&$ Hunt (2006) measured the coefficient of restitution for particle-particle collisions in a liquid and found a similar dependence on particle Stokes number. However, for 
Stokes numbers less than approximately 20 the authors observed that the target particle moved prior to impact due to the increase in hydrodynamic pressure as the impacting particle approached within a half a particle diameter.

Computational studies have also considered the problem of particle collisions in a liquid. By fixing the particle velocity at a constant value as it approaches a wall, Leweke, Thompson \& Hourigan (2004) computed the flow generated by a particle colliding normal to a surface without rebound; these simulations showed the development of vortex rings around the particle over a range of Reynolds numbers. In this simulation, a dynamic mesh moved with the sphere; as the sphere approached the wall, the mesh was refined. The sphere was stopped at a distance of 0.005 times the particle diameter from the wall to avoid the development of a mesh singularity as the particle impacted the surface; the particle was not allowed to rebound. TenCate et al. (2002) simulated a sphere settling toward a solid wall without rebound and compared with experiment results. Both the spatial structure and the temporal behaviour of the flow field were obtained. As the particle approached the wall, the authors included an explicit expression for the leading-order lubrication force, as previously suggested in the studies by Ladd (1997). Using a subgrid-scale lubrication force, Leweke et al.indicated that the particle approaches the wall in a smoother fashion than without the lubrication force (without the lubrication force, the particle motion is abruptly stopped when the surfaces contact); however, the lubrication force slows the particle so that the approach to the wall is longer than found in experiments. The authors only considered conditions in which there was no rebound.

Ardekani \& Rangel (2008) proposed a collision strategy that assumed no liquid is present between the two solid surfaces at collision and used the dry coefficient of restitution $e_{d}$ to calculate the instant rebound velocity directly when the distance between the surfaces becomes equal to the particle surface roughness height, $h_{\min }$, of order $1 \mu \mathrm{m}$. To resolve the flow at the length scale of $h_{\min }$, a finer mesh is used near the contact region; the simulation results depend on the mesh size and $h_{\min }$. Their model neglects the elastic deformation of the particles, which occurs over lengths comparable with the roughness height according to the elastohydrodynamics analysis in Davis et al. (1986). The authors do not compare their results with the trajectory of a bouncing particle; however, they do compare with experimental measurements of the speeds of the particle before and after collision through the effective coefficient of restitution data from Gondret et al. (2002). The recent work by Feng, Michaelides \& Mao (2010) presents a collision model using a spring and dashpot, similar to softsphere collision models for dry granular flows. Their analysis shows that the choice of the collision parameters (the constants in the spring and dashpot model) is critical to accurately predict experimental data.

The numerical studies by Al-Samieh \& Rahnejat (2002) calculated the pressure increase and surface deformation for a particle impacting a surface coated with a thin liquid layer. By coupling the equation for the motion of the liquid layer with the motion of the particle and the elasticity of the solid surfaces, the authors were able to calculate the pressure rise and compared it with the experiments by Safa \& Gohar (1986). For an impact at Stokes number of 15 (a $2.54 \mathrm{~mm}$ diameter steel sphere impacting a glass surface coated with a $30 \mu \mathrm{m}$ layer of oil with kinematic viscosity $0.43 \mathrm{~Pa} \mathrm{~s}$ at approximately $0.3 \mathrm{~m} \mathrm{~s}^{-1}$ ), the authors calculated pressure increases higher than found in the experiments (which was attributed to resolution issues in the experiments); the minimum film thickness was calculated as $2 \mu \mathrm{m}$ and a collision time of approximately $100 \mu \mathrm{s}$. 
This paper examines an individual particle collision in a viscous liquid and develops a computational collision model to enable advancements in numerical simulations of liquid-solid flows. To calculate the flow field an immersed boundary (IB) method is used to simulate the process of a rigid sphere settling and colliding with a nondeforming solid wall. By including a contact model, the numerical method captures the elasticity of the solid boundaries and mitigates the resolution problem when the particle is close to the wall. To validate the numerical method and calibrate the collision model, experiments on a steel sphere falling and colliding with a horizontal wall are performed and the trajectories of the sphere for multiple bounces are recorded. The numerical method and experiments are described in $\S \S 2$ and 3, respectively. Unlike earlier studies, the current work introduces a physically-based model that resolves the resolution issue when the particle approaches the wall and reproduces experimental measurements of a bouncing spheres over a wide range of Stokes numbers. A contact model accounting for the effects of the thin lubrication layer and solid elasticity is presented in $\S 4$. The simulation results are discussed in $\S 5$ and conclusions are drawn in $\S 6$.

\section{Numerical method}

\subsection{Fast immersed boundary projection method for the axisymmetric system}

This work obtains a solution for the incompressible constant-density flow field surrounding a moving immersed rigid sphere and a rigid stationary plane wall. The numerical method is of the finite-volume IB type and solves for the fluid vorticity and streamfunction on a regular Cartesian grid (with equally spaced cells of width and height, $\mathrm{d} x$ ), and utilizes a system of regularized forces defined at a set of Lagrangian points on the solid surfaces. The method is first-order accurate in space and secondorder accurate in time. The divergence-free constraint is automatically satisfied by the discretized streamfunction. The forces at the Lagrangian points distributed on the surface are found implicitly by requiring the no-slip boundary condition (as interpolated from the grid to the surface) to be satisfied. The resulting system of equations is solved using a projection method. Aside from no-slip conditions at the solid surfaces, the method implements an approximate treatment of the free-space boundary conditions at a finite distance from the sphere and plate. The computational cells are progressively coarsened away from the sphere and wall prior to closure with the approximate free-space boundary conditions. Except for modifications to handle the axisymmetric geometry, which are discussed below, full details and validation of the (planar flow) algorithm are presented in Taira \& Colonius (2007) and Colonius \& Taira (2008).

For the present study, the method is extended to axisymmetric geometries by reformulating the discrete finite-volume equations with the appropriate radial weighting terms in the definitions of gradient, curl, divergence, Laplacian, and the IB operators. With this modification, the cylindrical-coordinate version becomes algorithmically identical to the planar case, and the same fast algorithm may be employed. The detailed modifications to the spatial discretzation to account for the non-constant coordinate system matrices are described in $\mathrm{Li} \mathrm{(2010)}$ and, for brevity, are not repeated here.

The assumption of an axisymmetric flow field is computationally convenient, but limits the maximum Reynolds number, as symmetry-breaking disturbances are expected to occur at some critical Reynolds number. This value is not precisely known for the impacting sphere, but for steady flow over a sphere, numerical and experimental measurements by Johnson \& Patel (1999) give a critical Reynolds number of 210. For a sphere colliding with a wall, Thompson, Leweke \& Hourigan 
(2007) showed that when the running distance is short $(L / D \leqslant 7.5$, where $L$ is the initial distance between the sphere and the wall), the sphere wake is not fully developed; hence, the flow is in a transient axisymmetric state and does not exhibit asymmetry in the form of vortex-loop shedding, which occurs (asymptotically) at Reynolds number above 210. In the current work, the running distance is less than 7 and the particle Reynolds number is less than 250 so that the assumption of an axisymmetric flow field appears to be justified.

A three-level computational domain is used in the current simulation with uniform grid size $\mathrm{d} z=\mathrm{d} r=2^{i-1} 0.01 D$ for the $i$ th-level domain (for detailed information about the multi-domain technique, see Colonius \& Taira 2008). A far-field boundary condition (slip but non-penetrating) is applied at the boundary of the third-level domain as required by the numerical method. For the symmetric axis $r=0$, the boundary condition is strictly satisfied. For the other three boundaries, the slip-nonpenetrating boundary condition for the large domain with coarser mesh is appropriate since the real container boundary is far away and in accordance with the third domain.

To validate the cylindrical-coordinate modifications, the simulation results for the flow over a stationary sphere were found on a series of grids with increasing resolution until convergence occurred and then were compared with previously published results (Taira \& Colonius 2007; Johnson \& Patel 1999). The drag coefficient for a flow with Reynolds number 100 is 1.10 , and the reported values are 1.14 and 1.10 . For a flow with Reynolds number 200, the drag coefficient is 0.78 and the two earlier studies report a value of 0.80 .

For the case of a moving sphere, the time-marching scheme of the original IB projection method was also modified to treat the two-way coupled system where the motion of the Lagrangian points on the surface are found by integrating an equation of motion for the sphere; the details are discussed in $\S 2.2$, and validations for the two-way coupled system are presented in $\S 2.3$, where we compare with corresponding experiments and simulations of TenCate et al. (2002).

\subsection{The evolution of the flow coupled to the motion of the particle}

The flow field evolution and the motion of the sphere are coupled by writing Newton's law in the vertical direction for the sphere:

$$
m_{p} \frac{\mathrm{d} V}{\mathrm{~d} t}=f_{\text {sim }}+m_{p} g+f_{b},
$$

where $V$ is the velocity of the sphere which is considered to be a uniform value over the whole sphere surface; $m_{p}$ is the mass of the particle and $g$ is the gravitational acceleration; $f_{b}$ is the vertical buoyancy force and $f_{\text {sim }}$ is the liquid-solid interaction force in the vertical direction calculated from the solution of the Navier-Stokes equations. The position and the velocity of the sphere are the boundary conditions for the Navier-Stokes equations. The coupled evolution of the flow field and the motion of the sphere are integrated in time using a second-order Runge-Kutta method.

\subsection{Validation of the numerical method}

To validate the coupled algorithm, the simulation results are compared with the experimental results by TenCate et al. (2002). In the experiment, the trajectory and associated flow field of a settling sphere are measured from the moment of release until rest at the bottom of a vessel. The Reynolds number based on the terminal velocity is 31.9 . The density of the sphere is $1.12 \times 10^{3} \mathrm{~kg} \mathrm{~m}^{-3}$ and the density of the liquid is $0.96 \times 10^{3} \mathrm{~kg} \mathrm{~m}^{-3}$. Simulations with the above input parameters were performed. 
(a)
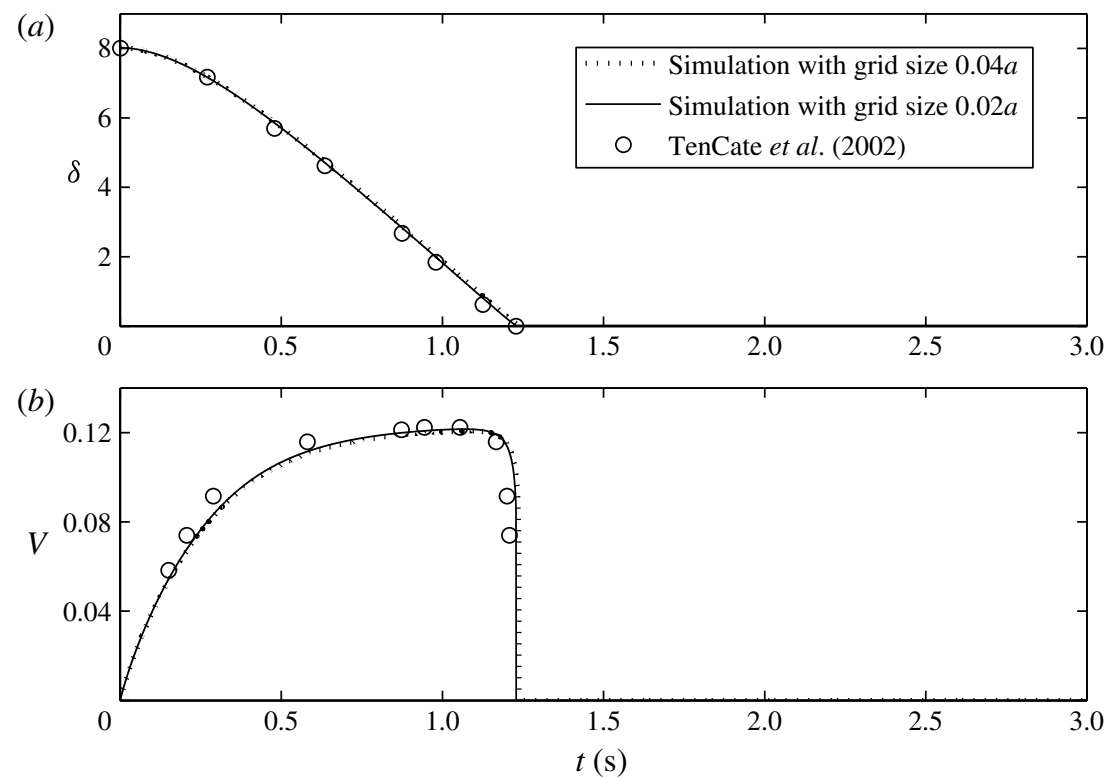

FIgURE 1. Comparison of $(a)$ trajectory and $(b)$ velocity profiles for the experiments of TenCate et al. (2002), and the current simulations for a sphere falling on to a surface; the Stokes number based on the settling velocity is 4.1. $a$ is the radius of the sphere.

The trajectory and the velocity profiles are compared in figure 1 . The circles in the figure are digitized from figure 8 in TenCate et al. (2002). The comparison shows that the simulation coupling the evolution of the flow field and the motion of the sphere predicts the same motion of the sphere as found in the experiment. Note that the Stokes number at impact $(S t=4.1)$ is small and the particle does not rebound from the wall. In the current simulations, similar results were computed with two different grid sizes in order to establish convergence.

Figure 2 compares the flow field at the moment when the sphere is a halfdiameter from the wall with the particle image velocimetry (PIV) measurement of TenCate et al. (2002). The velocity contours above the sphere are not included in the experimental result. The velocity magnitude contours show a good agreement between the experimental and the simulated flow field.

Time series of the fluid velocity at a particular point in the flow domain are compared in figure 3 . The point is one diameter from the wall and one diameter from the centre of the sphere. The radial and axial velocity components are given on the upper and lower plots, respectively. The evolution of the velocity components shows that a vortex passes the point and the wake of the sphere follows after the sphere comes to rest. The good agreement indicates that the numerical method accurately simulates not only the dynamic behaviour of the sphere but also the evolution of the flow field.

\section{Experiments}

The experiments by TenCate et al. (2002) did not include results for higher Stokes numbers. When the particle Stokes number increases, the particle rebounds after 

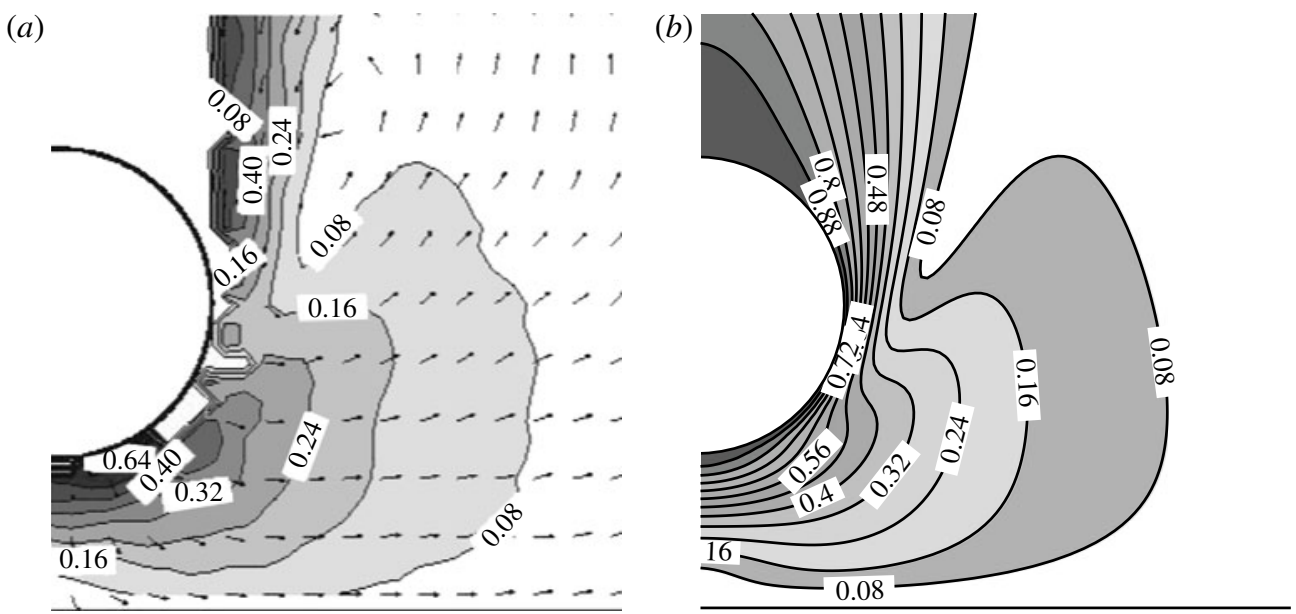

Figure 2. Comparison of (a) the PIV experiment data from TenCate et al. (2002) and (b) the simulated result of flow field when the sphere is half a sphere diameter from the wall. Contours indicate the normalized velocity magnitude. The blank space above the sphere in (a) results from the limitations of the experimental method.

collision. The experiments by Gondret et al. (2002) include particle rebound but do not include the initial trajectory of the particle after it is released. To provide data with which to compare the simulations, new experiments are conducted where the particle is released from rest in a quiescent tank; the particle collides with the lower wall and rebounds several times. As a result, the experimental data set includes the interaction of the particle and liquid as the particle accelerates and the particle-wall collision process.

\subsection{Experiment apparatus}

The experiment setup is shown schematically in figure 4. The experiments were performed in a glass rectangular tank with length $\times$ width $\times$ height of $600 \mathrm{~mm} \times 350 \mathrm{~mm} \times 450 \mathrm{~mm}$ that contains a mixture of glycerol and water. A steel sphere with diameter $9.5 \mathrm{~mm}$ and surface roughness of $0.02 \mu \mathrm{m}$ is dropped from an electromagnetic release mechanism that is fixed on the top of the tank. A trigger pad is used to cut off the current in the electromagnetic mechanism, releasing the steel sphere from zero velocity under quiescent ambient fluid conditions. The circular release surface with diameter $52 \mathrm{~mm}$ is immersed to a certain depth $(>50 \mathrm{~mm})$ under the free surface of the liquid, and the effect of the liquid free surface on the experiment is ignored. A cylinder-shaped Zerodur block with diameter $150 \mathrm{~mm}$ and height $100 \mathrm{~mm}$ is placed coaxially a certain distance below the release mechanism as a target wall. Zerodur is a hard glass-like material and the block is polished to minimize the effect of wall roughness (roughness height of $0.02 \mu \mathrm{m}$ ). The sphere is dropped from the centre of the release surface and falls along the axis of the Zerodur block. The dimension of the glass tank is large compared with the sphere diameter so that the flow field around the moving sphere can be considered as axisymmetric as long as the particle Reynolds number is less than 250.

A high-speed videography system (a high-speed camera, a control/display monitor and a record trigger) captures the particle dynamic behaviour. Figure 5 shows an example image with $h$ as the gap between the sphere and the wall. The camera frame 

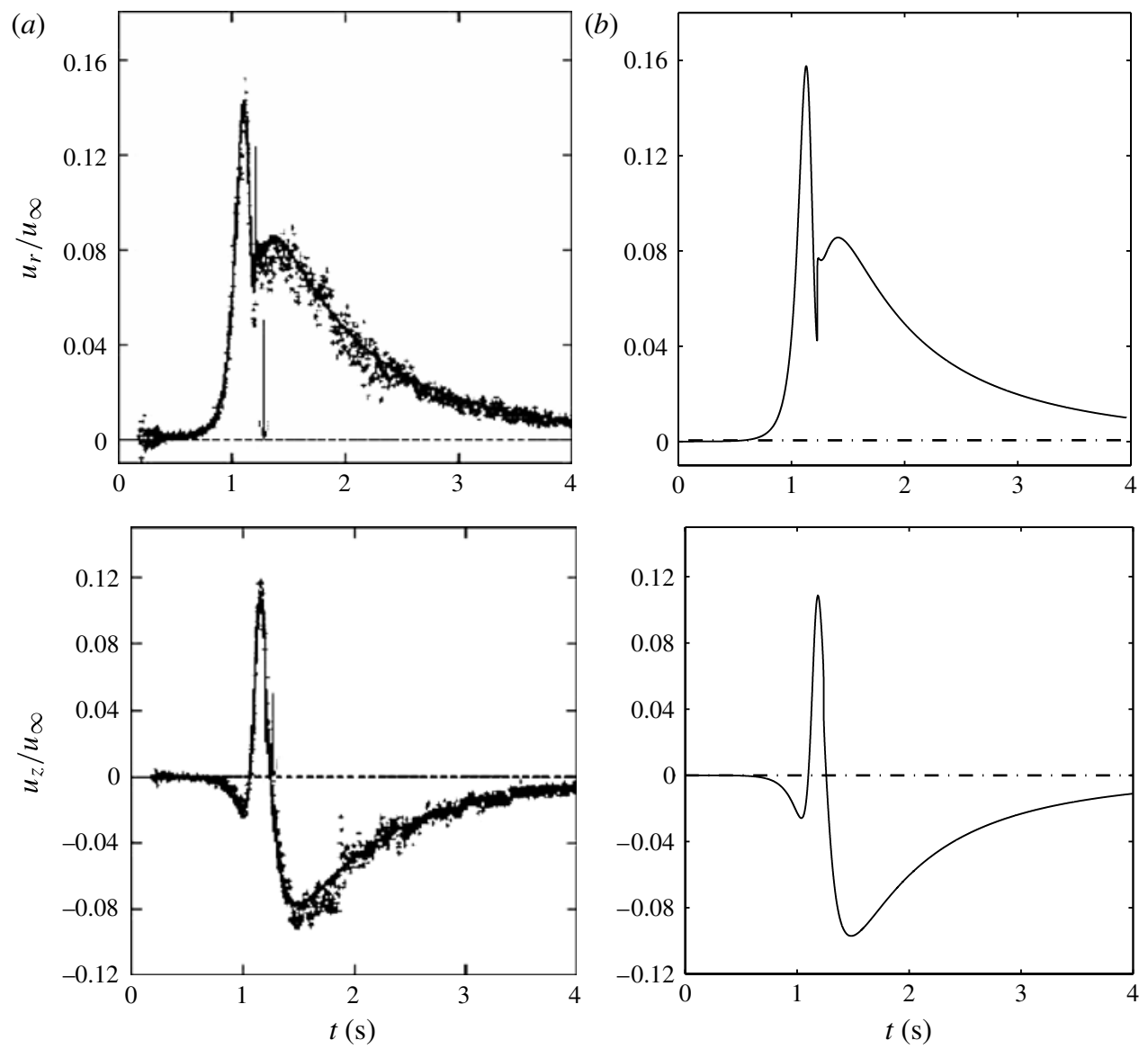

FIGURE 3. Comparison of time series of the radial and axial fluid velocity at a point located one diameter from the wall and one diameter from the centre of the sphere. $(a)$ The experimental result and the lattice Boltzmann simulation from TenCate et al. (2002) and (b) the results from the current simulation.

rate is 4000 frames per second so that the time interval between two successive frames is $0.25 \mathrm{~ms}$. Under this frame rate, the resolution is 160 pixels wide by 140 pixels high. More details of the motion of a particle, especially when it is about to collide with the wall, can be obtained by using this high frame rate.

\subsection{Material properties}

The properties of the solid material are listed in table 1, including solid density, $\rho_{p}$, Young's modulus, $E$, Poisson's ratio, $v$. The experiments used aqueous glycerol solutions, which allows a large range of viscosities by changing the mixture proportions. Because the viscosity of the mixture varies with temperature, the liquid temperature is measured before each collision by a digital thermocouple thermometer. The apparent specific gravity of the liquid is measured by a hydrometer. Based on the measured temperature and apparent specific gravity, the concentration, density and viscosity of the mixture can be found from Dow Chemical Synthetic Glycerin Products. A typical value of the apparent specific gravity is 1.205 at $20^{\circ} \mathrm{C}$ corresponding to a density of $1203 \mathrm{~kg} \mathrm{~m}^{-3}$ and a viscosity of $50.2 \times 10^{-3} \mathrm{~Pa} \mathrm{~s}$. 


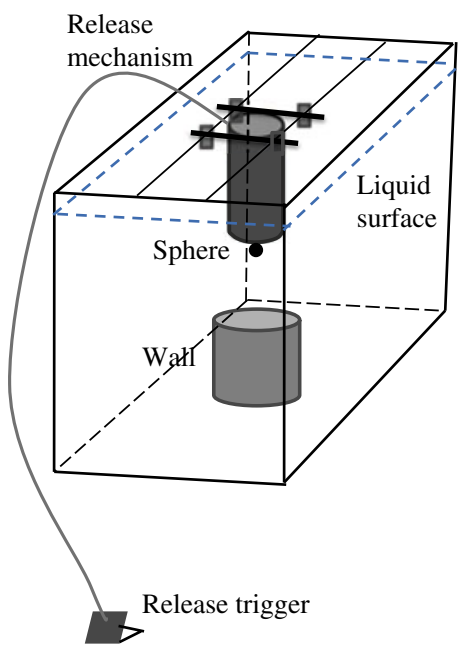

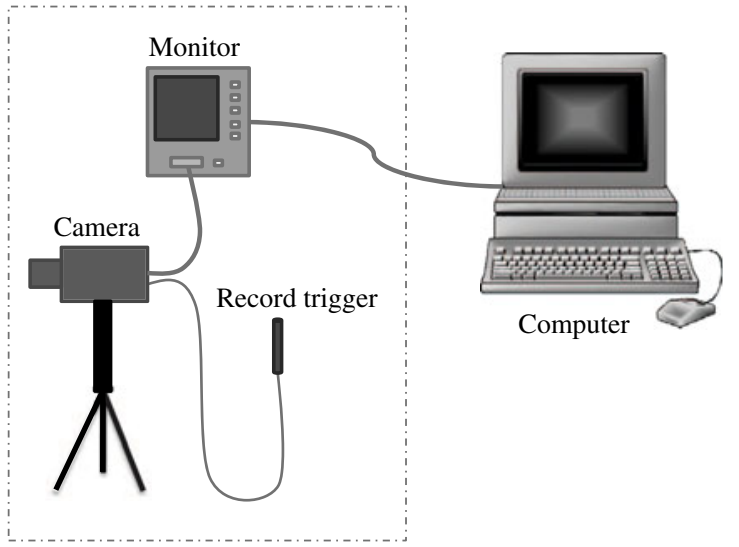

High speed videography

FIGURE 4. Schematic of the experiment setup.

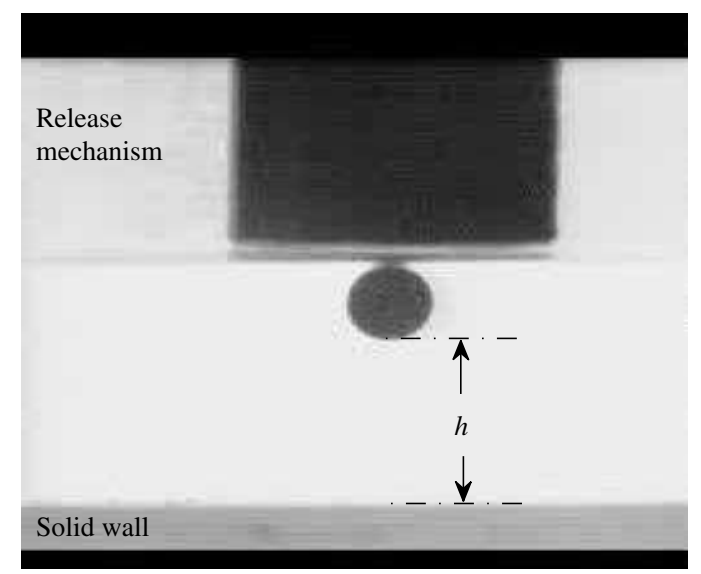

FIGURE 5. The image shown on the control/display monitor. $h$ is the gap between the sphere and the wall.

$\begin{array}{lccc}\text { Material } & \rho_{p}\left(\mathrm{~kg} \mathrm{~m}^{-3}\right) & E(\mathrm{GPa}) & v \\ \text { Steel } & 7780 & 200 & 0.33 \\ \text { Zerodur } & 2530 & 91 & 0.24\end{array}$

TABLE 1. Properties of the sphere and the wall used in the collision experiments.

\subsection{Experimental results}

Collisions with different impact Stokes numbers were obtained by placing the wall at different distances from the release surface. After applying a template matching method, the trajectory and velocity of a sphere are obtained for each collision from 


$\begin{array}{lccccc}\text { Case } & h(t=0)(\mathrm{mm}) & h(t=0) / d & V_{i}\left(\mathrm{~m} \mathrm{~s}^{-1}\right) & R e_{I} & R e_{I I} \\ 1 & 5.5 & 0.58 & 0.231 & 53 & 29 \\ 2 & 10.5 & 1.10 & 0.327 & 75 & 41 \\ 3 & 15.2 & 1.60 & 0.393 & 90 & 55 \\ 4 & 19.6 & 2.06 & 0.449 & 104 & 60 \\ 5 & 25.3 & 2.66 & 0.484 & 113 & 67 \\ 6 & 28.4 & 2.98 & 0.506 & 123 & 68 \\ 7 & 35.7 & 3.75 & 0.554 & 127 & 69\end{array}$

TABLE 2. Experiments with particle Reynolds numbers at first impact $\left(R e_{I}\right)$ and second impact $\left(R e_{I I}\right)$. Every Reynolds number is an average value from the results of three experiments repeated at the same initial distance.

the recorded videos. The impact Reynolds number is calculated based on the impact velocity of the sphere, $V_{i}$, by averaging the measured speed over the $0.002 \mathrm{~s}$ before collision since Joseph et al. (2001) and Gondret et al. (2002) showed that the impact speed was not changing within this time interval in their experiments. Because of the small values of $h(t=0) / D$, the particle does not achieve its terminal velocity before it contacts the wall. Thus, the particle Reynolds number is related to the fall distance.

A list of the experimental cases and associated parameters is given in table 2 . The trajectories for different drop heights are compared in figure 6. Ideally, the initial trajectories for each case should coincide. The deviation provides a measure of the uncertainty of the experiments; the maximum error is less than $3 \%$. In all the cases, the particle bounced at least twice. The maximum height achieved in the rebound motion is lower than the initial height, which is the result of viscous losses and inelasticity. For the conditions of the current experiments, the impact velocities are all lower than the speed at which yield occurs in stainless steel (approximately $4.6 \mathrm{~m} \mathrm{~s}^{-1}$ based on work by Ruiz-Angulo \& Hunt 2010).

\section{Contact model}

\subsection{Physics of a lubricated impact of a sphere on a wall}

As noted in the introduction, several numerical studies have introduced methods to capture the physics of a particle-particle or particle-wall collision. In a collision, the lubrication layer between the colliding surface can be thin (less than a micron) and the collision time can be on the order of tens of microseconds; surface deformation and particle roughness further complicate the collision process. Hence, this gap region is difficult to resolve in a numerical simulation and a finer grid can delay but not prevent the problem. In the current simulations, the bodies are rigid; hence, the elasticity of the material is not included. As a result, the simulations do not include the energy stored in the elastic deformation of the sphere and the wall, which is critical to the rebounding of an impacting sphere. Instead of solving the thin lubrication layer and the deformation of the solid parts, a contact model is developed to capture the collision process: the effect of the liquid layer is incorporated by using a liquid-solid interaction force term; the kinetic energy stored in the deformation of the solid bodies is included in an elastic force that acts on the falling sphere. 


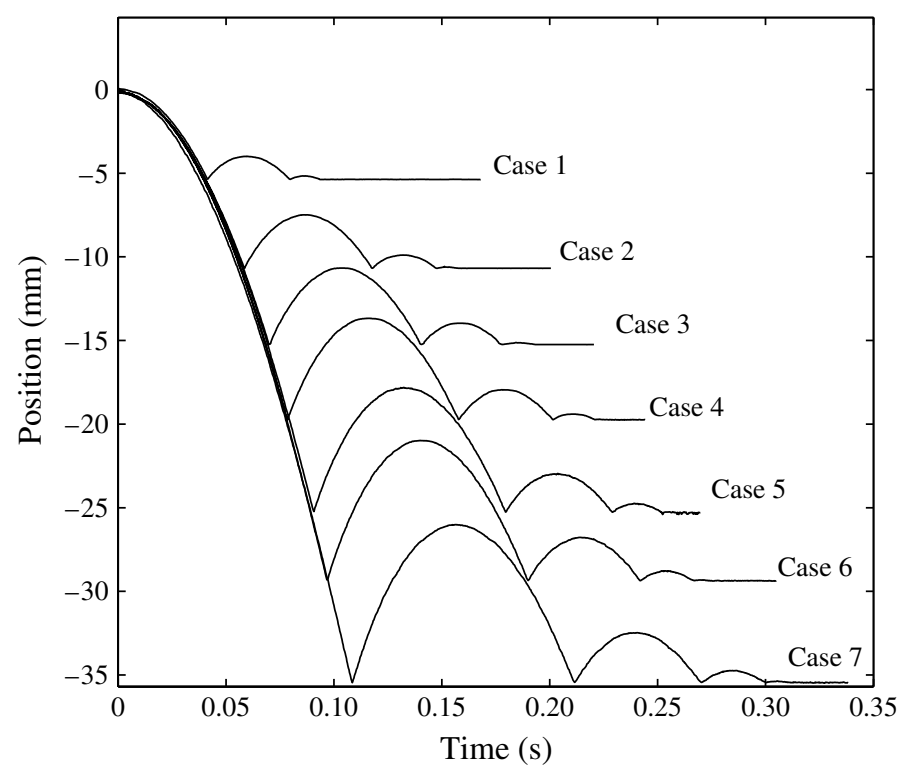

FIGURE 6. Particle trajectories for the cases described in table 2.

\subsection{Liquid-solid interaction force with wall effect}

The interaction between a moving solid particle and the surrounding fluid has been studied by different researchers. A thorough review can be found in Michaelide (1997). Three types of hydrodynamic forces, namely the Stokes drag force, the added mass force and the history force, are most widely discussed and established. When there is an additional solid boundary, the hydrodynamic forces are modified because the fluid is restricted by the second solid boundary. Prior studies have developed correction terms for the gap between the two solid boundaries for the three forces. Yang (2006) simplified the analytic formulae for the hydrodynamic forces to include the wall and higher Reynolds number effects. The formulae that were validated with their experimental results are represented below for $f_{D}$, Stokes drag force; $f_{H}$, the history force; and $f_{A M}$, the added mass force:

$$
\begin{gathered}
f_{D}=-6 \pi \mu a V \lambda(\delta, R e), \\
f_{H}=-6 \pi \mu a K_{H}(\delta)^{3 / 2} \int_{0}^{t} \frac{\mathrm{d} V}{\mathrm{~d} \tau} K(t-\tau) \mathrm{d} \tau, \\
f_{A M}=-\frac{1}{2} m_{l}[1+3 W(\delta)] \frac{\mathrm{d} V}{\mathrm{~d} t}-\frac{3}{4} m_{l} V \frac{\mathrm{d} W}{\mathrm{~d} t}
\end{gathered}
$$

where $\delta=h / D$ is the non-dimensional gap between the particle and the wall, $a$ is the radius of the particle and $m_{l}$ is the liquid mass. $K_{H}(\delta)$ and $W(\delta)$ are coefficients that depend on the gap between the particle and the wall $\lambda(\delta, R e)$ is a near-wall correction term based on both the gap width and the particle Reynolds number; $K(t-\tau)$ is a time kernel that describes the local dissipation mechanism and diminishes the effect of the history force due to the earlier sphere acceleration (see Kim, Elghobashi \& Sirignano 1998). The added mass force accounts for the dynamic pressure distribution around the particle; the history force results from the temporal delay in the boundary layer development over the unsteady sphere surface and is an accumulated effect of the acceleration history of the particle. These formulae are valid only in the region near 


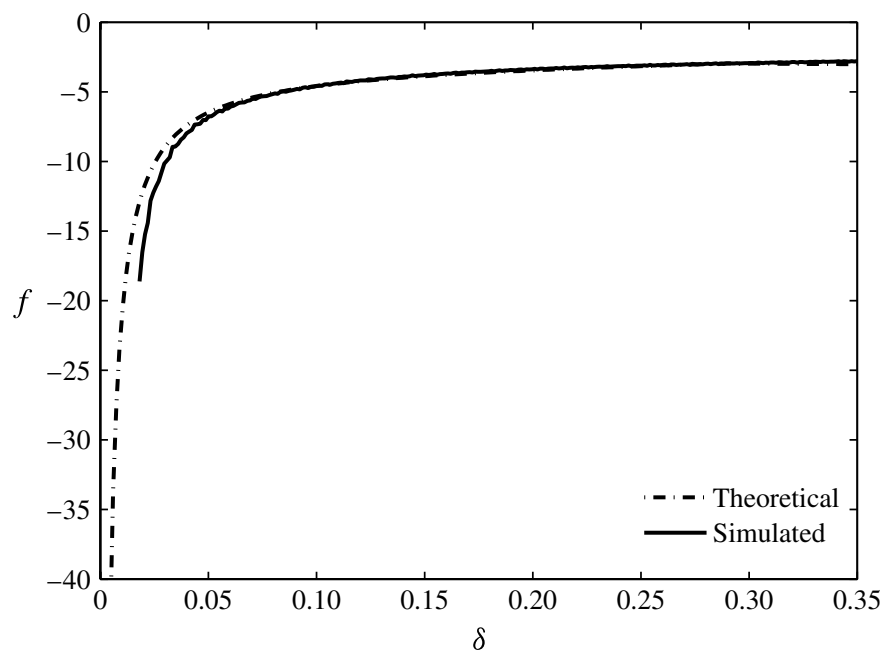

FIGURE 7. The liquid-solid interaction force for an impact process. The theoretical force, $f_{t h r}$, is calculated from Yang (2006)'s formulae with the measured sphere kinetic data from case 3 . Force $f_{\text {sim }}$ is the simulated result when prescribing the motion of the sphere with the trajectory measured in case 3 . The force is non-dimensionalized by $(4 / 3) \pi a^{3} \rho_{l} g$.

the wall. The sum of these three forces is defined as the total hydrodynamic effect on the solid sphere, $f_{t h r}$

$$
f_{\text {thr }}=f_{D}+f_{H}+f_{A M} .
$$

When the gap decreases to zero, the formulae converge to the classical lubrication theory results.

Using the falling trajectory measured from the current experiments, the hydrodynamic force calculated from (4.4), $f_{t h r}$, is found to overlap with $f_{\text {sim }}$, the simulated result when applying the numerical method described in $\S 2$. Figure 7 is a typical plot from a single run, which shows the hydrodynamic force as a function of the gap between the sphere and the wall in experimental case 3. The good agreement for the gap ranging from 0.1 to 0.35 shows that Yang's (2006) formulae could be used to compute the force as the sphere approaches the wall, especially when the simulation becomes unreliable at very small gaps $(<0.05)$ because of the resolution problem.

To resolve the flow when the gap is small, a liquid-solid interaction model is proposed that blends the simulated and the theoretical forces when the gap decreases below a threshold value, $\delta_{s l}$. The liquid-solid force, $f_{s l}$, is computed as

$$
f_{s l}=H\left(\frac{\delta}{\delta_{s l}}\right) f_{t h r}+\left[1-H\left(\frac{\delta}{\delta_{s l}}\right)\right] f_{\text {sim }}
$$

where

$$
H\left(\frac{\delta}{\delta_{s l}}\right)=\frac{1}{1+\mathrm{e}^{10\left(\left(\delta / \delta_{s l}\right)-1\right)}}
$$

is a smoothed Heaviside function, as shown in figure $8(a)$. The function was chosen to smoothly transfer the hydrodynamic force from the simulated value to the theoretical value as the gap diminishes. Based on figure $7, \delta_{s l}$ is conservatively taken as 0.2 . Clearly, the necessary value of $\delta_{s l}$ depends on the grid resolution of the fluid 

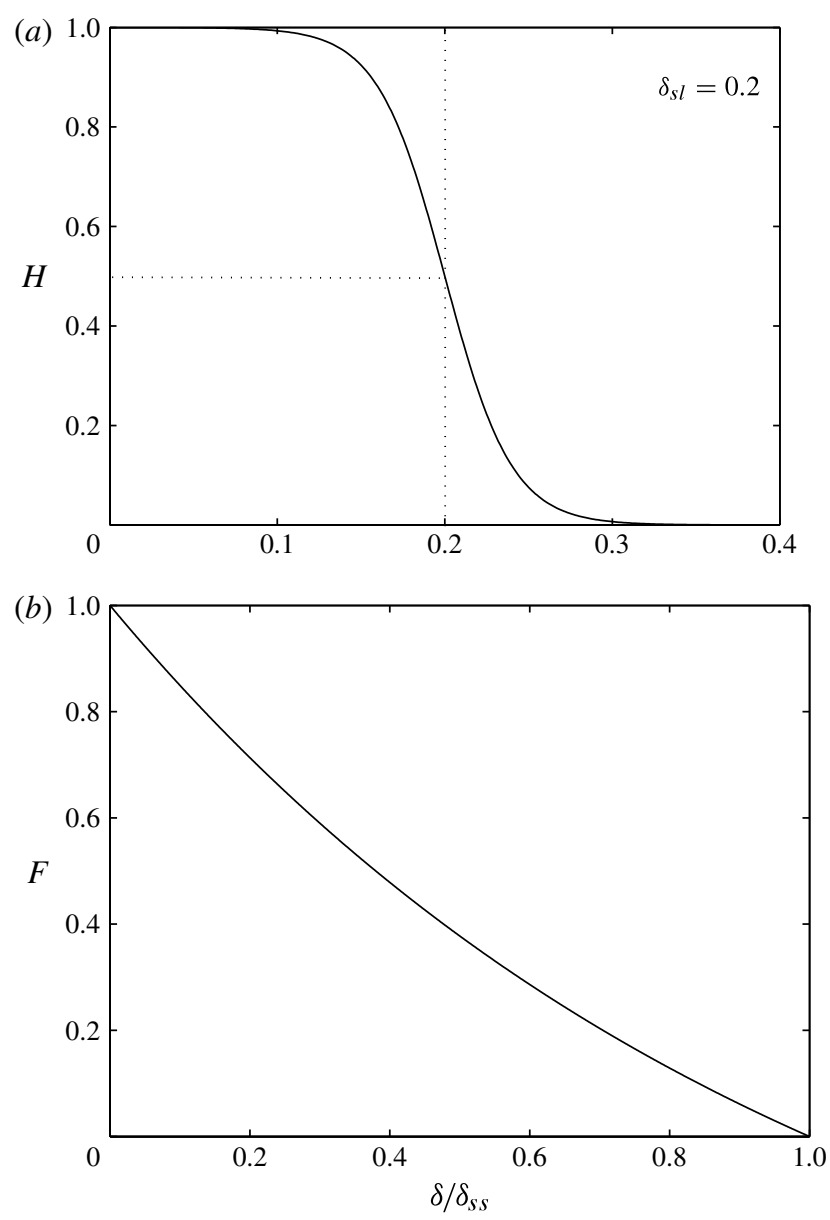

FIGURE 8. (a) Function $H$ for $\delta_{s l}=0.2$. (b) Function $F$.

simulation. The finer the grid, the smaller the value of $\delta_{s l}$ that could be used. On the other hand, it seems reasonable to fix it at a conservative value independent of the grid resolution.

\subsection{Elastic force}

Based on Ruiz-Angulo \& Hunt (2010), when a particle impacts with a velocity lower than the yield velocity, the collision is within the elastic or nearly elastic regime. According to the Hertz elastic theory (see Timoshenko \& Goodier 1970) for a collision without interstitial fluid effects, the elastic force, $W_{o}$, is calculated as

$$
W_{o}=\frac{4}{3} a^{2} E^{*}\left(\frac{5 \pi}{4 E^{*}} \rho_{p} V_{I}^{2}\right)^{3 / 5}
$$

where $V_{I}$ is the particle impact velocity and $E^{*}=\left[\left(1-v_{1}^{2}\right) / E_{1}+\left(1-v_{2}^{2}\right) / E_{2}\right]^{-1}$ is the reduced modulus based on the Young's modulus, $E$, and the Poisson ratio, $v$, for the 
two materials. The proposed elastic force term is based on this Hertz elastic force:

$$
f_{s s}=F\left(\frac{\delta}{\delta_{s s}}\right) e_{d} W_{o},
$$

where the dry coefficient of restitution $e_{d}$ is included to take into account the inelasticity of the particle. In the current experiments, inelasticity effects are not significant and the value of $e_{d}$ is taken as a constant equal to 0.97. Different forms of the tuning function $F\left(\delta / \delta_{s s}\right)$ had been tried to gradually introduce the elastic effect based on the solid-solid interaction. After comparing with the experimental results, the function $F$ is given the following form:

$$
F\left(\frac{\delta}{\delta_{s s}}\right)= \begin{cases}\frac{\mathrm{e}^{(-\delta / \delta s s)}-\mathrm{e}^{-1}}{1-\mathrm{e}^{-1}}, & 0 \leqslant \delta \leqslant \delta_{s s} \\ 0, & \delta>\delta_{s s} .\end{cases}
$$

and is shown in figure $8(b)$. When the gap is large, there is no elastic force; however, when the gap decreases and approaches zero, the function $F\left(\delta / \delta_{s s}\right)$ increases to a value of unity and the elastic effect $f_{s s}$ increases to the Hertzian force evaluated at the impact speed.

The parameter $\delta_{s s}$ fixes the distance from the wall at which the particle elasticity becomes important; the physical meaning of $\delta_{s s}$ is also explained by employing the concept of 'effective radius'. As a sphere approaches the wall, the hydrodynamic pressure increases in the fluid layer between the two solid surfaces; with the increased pressure, the surfaces deform and collision happens either through the layer of fluid between the surfaces or between the roughness elements (see Davis et al. 1986; Barnocky \& Davis 1989; Joseph et al. 2001). In addition as the fluid is compressed, its density and viscosity may increases, which may further limit the approach of the surfaces. Thus, the approaching sphere can be considered to have a virtual radius $a^{\prime}=a+\delta_{s s}$ larger than the physical value $a$. When the gap between the sphere and the target wall decreases to $\delta_{s s}$, the virtual sphere with the effective radius begins to reach the wall. A similar concept was employed by Nguyen \& Ladd (2002) who proposed a hydrodynamic radius for particles approaching a solid boundary to account for the lubrication layer effect; in their simulations the hydrodynamics radius was $a_{h y}=a+\Delta$ and the value of $\Delta$ varied from 0 to $0.05 a$ depending on the viscosity of the fluid and the particle radius $a$.

\subsection{Equation of motion with the contact model}

Including the above forces, the final equation of motion in the vertical direction with the contact model is

$$
m_{p} \frac{\mathrm{d} V}{\mathrm{~d} t}=f_{s l}+f_{s s}+m_{p} g+f_{b},
$$

By using (4.10) instead of (2.1) to couple with the flow field, the motion of the sphere both before and after the collision process can be computed with known input parameters based on the initial distance, the gravitational acceleration, the solid-liquid density ratio, the diameter of the sphere, and the viscosity of the liquid. Without the elastic force term (as given in (4.10)), the particle would not be able to rebound from the wall.

To calibrate the value of $\delta_{s s}$, the computed particle trajectories from the simulations of case 3 for different values of $\delta_{s s}$ are compared with the averaged experimental 


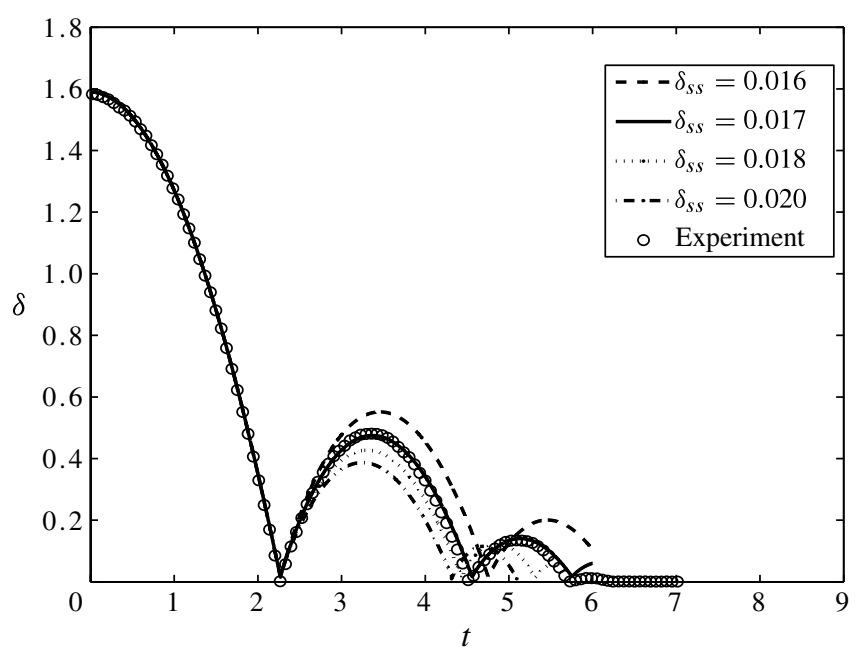

FIGURE 9. Trajectory of the sphere in case 3.

trajectory, as shown in figure 9. The horizontal axis represents the dimensionless time, $t$, which is the actual time divided by $\sqrt{D / g}$. Figure 9 shows that a greater value of $\delta_{s s}$ results in a lower maximum rebound height. The simulation using $\delta_{s s}=0.017$ gives the best fit for the first two rebounds. Thus this value is used for the contact model. The results for collisions in other cases and sensitivity of the results to variations in $\delta_{s s}$ are discussed in the following section.

\section{Results and discussion}

\subsection{Particle velocity}

Figure 10 shows the particle velocity in case 3 as a function of the gap between the particle and the wall. The velocity has been non-dimensionalized by $\sqrt{D g}$. In figure $10(a)$, the particle starts to fall from $\delta=1.6$ with zero velocity. The velocity increases as the gap decreases. The dashed line is an analytical result obtained by solving the equation

$$
m_{p} \frac{\mathrm{d} V}{\mathrm{~d} t}=\left(m_{p}-m_{l}\right) g,
$$

when only the gravitational and buoyancy forces are considered for an isolated sphere under the same initial conditions and a dry coefficient of restitution is applied. Thus, the difference between the solid line and the dashed line shows the hydrodynamic effect of the surrounding liquid on the motion of the particle. Figure $10(b)$ shows the enlarged details when the particle is about to collide with the wall. The impact velocity begins to decrease well before $\delta$ reaches $\delta_{s s}$ where the elastic force starts to take effect. This decrease in velocity results from the hydrodynamic force that increases sharply as the gap, $\delta$, diminishes. The particle decelerates before it rebounds. This deceleration prior to impact was also observed in the experiments of Joseph et al. (2001) and the simulations of Ardekani \& Rangel (2008). As the gap decreases to less than $\delta_{s s}$, the elastic force stops the approach of the particle. The rebound velocity has a sudden decrease after $\delta>\delta_{s s}$ as shown in figure 10(c) because the elastic force falls to zero and the hydrodynamic forces increase the viscous dissipation as more surrounding liquid re-enters the growing gap between the particle and the wall. 

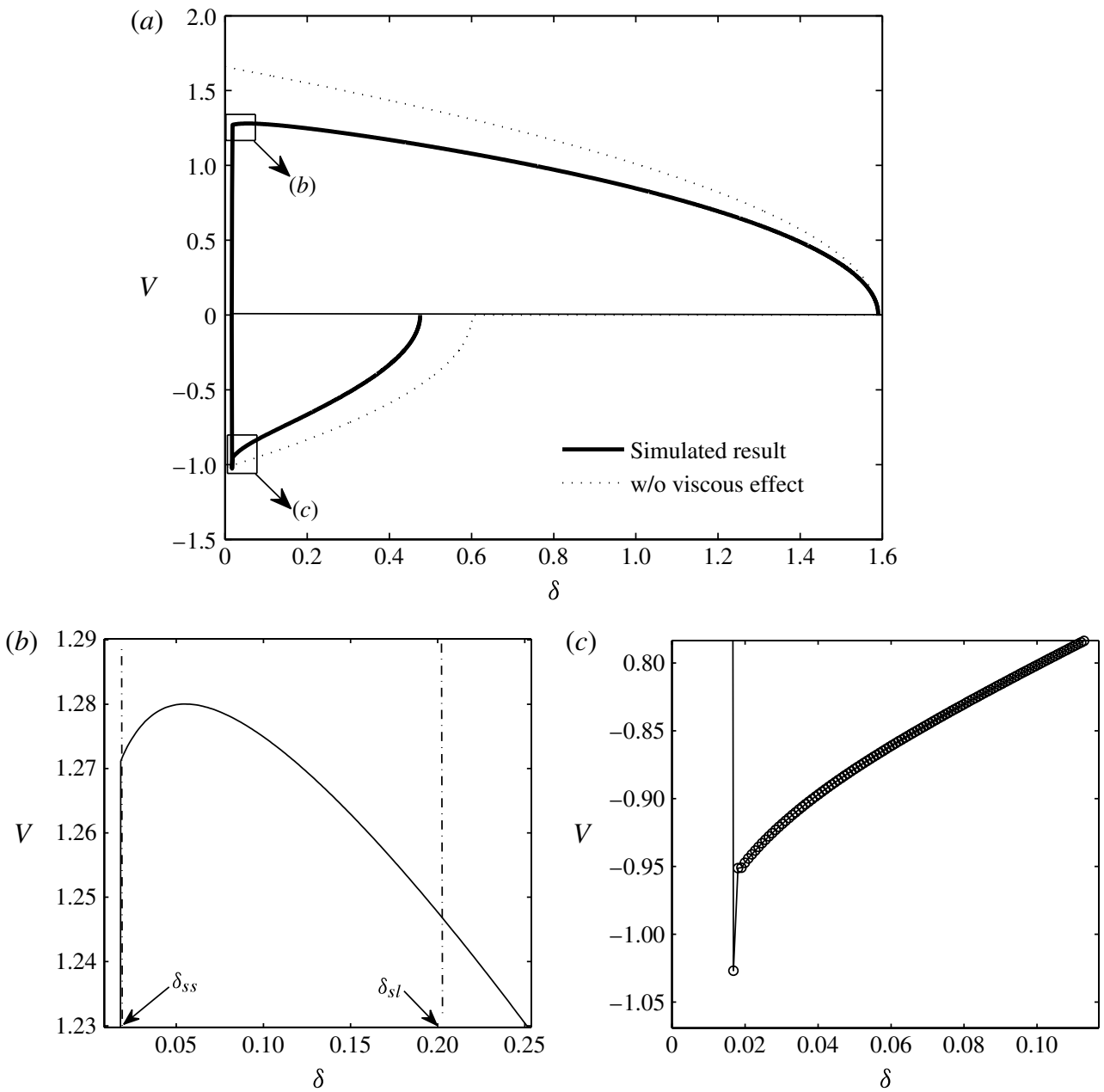

FIGURE 10. Velocity of the incoming and outgoing particle as a function of the gap between the particle and the wall. The solid line is the simulated result for case 3 . The dotted line is the analytical result when considering gravity and the buoyancy force on the particle and no hydrodynamic force. $(b),(c)$ Enlarged details of the portions of $(a)$ circumscribed in black rectangular boxes.

\subsection{Qualitative flow features}

The numerical simulation with the contact model not only captures the dynamics of the particles but also presents the evolution of the surrounding flow field during the falling and rebounding process. For the experimental case 7, figure $11(b)$ shows snapshots of the vorticity field of the flow around the sphere at different times corresponding to the points on the trajectory in figure 11(a). The particle Reynolds number of the first impact is 134 . Unlike the study of vorticity dynamics in Thompson et al. (2007) that prescribed the velocity of the sphere at a constant value and did not include the rebound, the current simulation solves the vorticity field when the sphere accelerates, decelerates and reverses directions. The first snapshot shows the primary vortex ring generated from the wake vorticity and the opposite-sign vorticity generated at the wall when the particle is just about to collide. The second snapshot is taken during the rebound after the first collision. The vorticity of opposite sign 

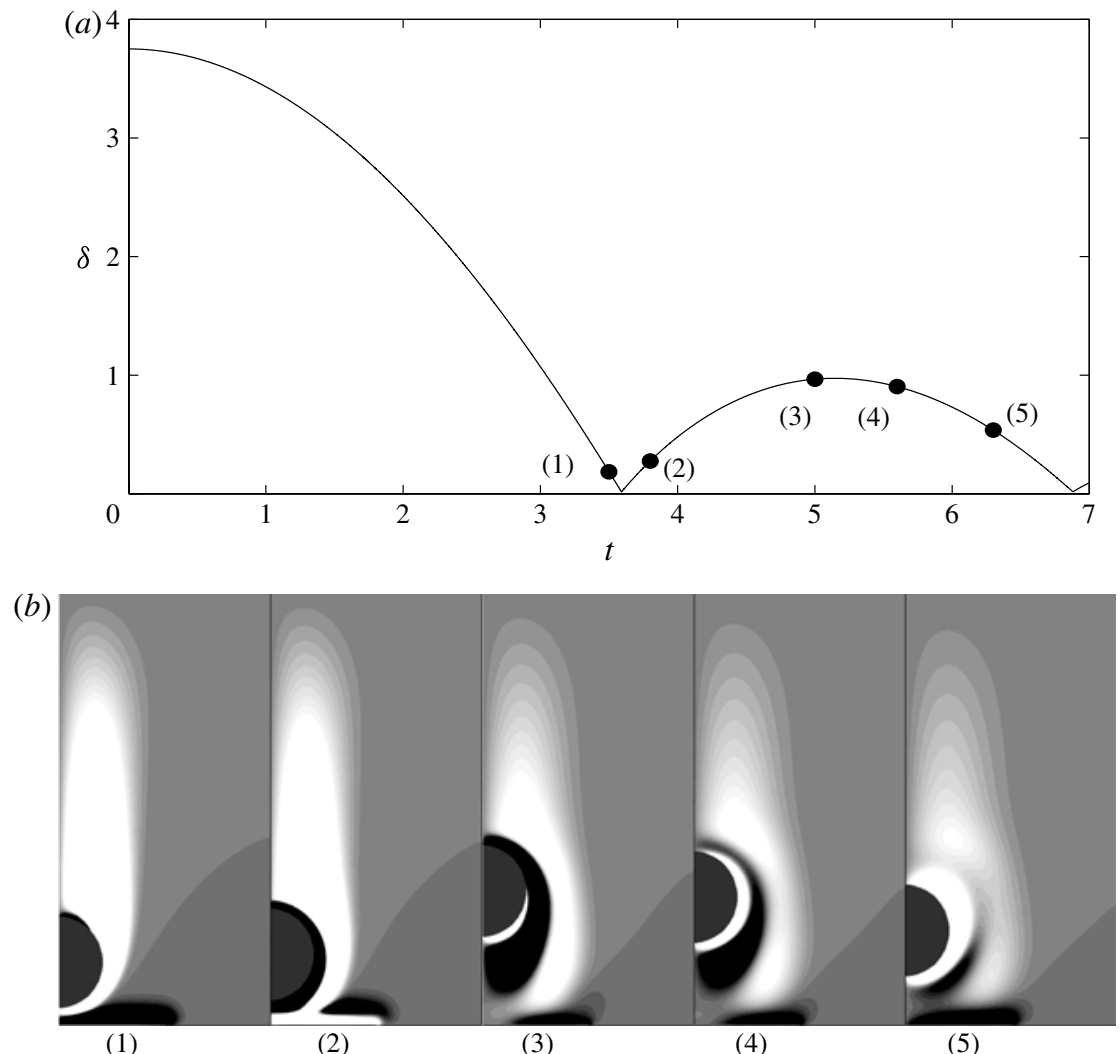

FIGURE 11. Snapshots of the vorticity field around the sphere at different times. Contour levels from -0.5 to 0.5 in increments of 0.05 are chosen for a good depiction of the weak vorticity field structure. The black semicircular area shows the location of the sphere: (a) particle trajectory; (b) vorticity field.

is generated at the sphere surface as the rebounding sphere moves upwards through the primary wake ring to form a secondary vortex ring. Near the wall, the new vorticity forms as the result of the liquid re-entering the gap. As the sphere continues moving upwards, the primary vorticity is stretched and expanded as the sphere passes. A vortex-ring dipole forms from the combination of the primary and the secondary vorticity structures. The new positive vorticity attached to the bottom of the sphere shown in the third snapshot is formed because the sphere's velocity decreases to zero as it approaches the maximum height of the first rebound. When the sphere starts to fall again, the attached vorticity becomes a new stronger primary ring. The secondary vorticity is slowly dissipated by the surrounding opposite-sign vortices and the original primary vortex ring propagates radially before being stretched and merged with the new primary vorticity, as shown in the fourth and the fifth snapshots. The complex vorticity structure entangles the particle and dissipates part of its kinetic energy.

\subsection{Validation of the contact model}

When the proposed contact model with $\delta_{s s}=0.017$ is applied to the collision processes with different Stokes numbers in the experimental cases 1-7 listed in table 2, the trajectories calculated from the simulation show good agreement with the experimental results for most of the cases. To estimate the deviation, a relative error defined as 

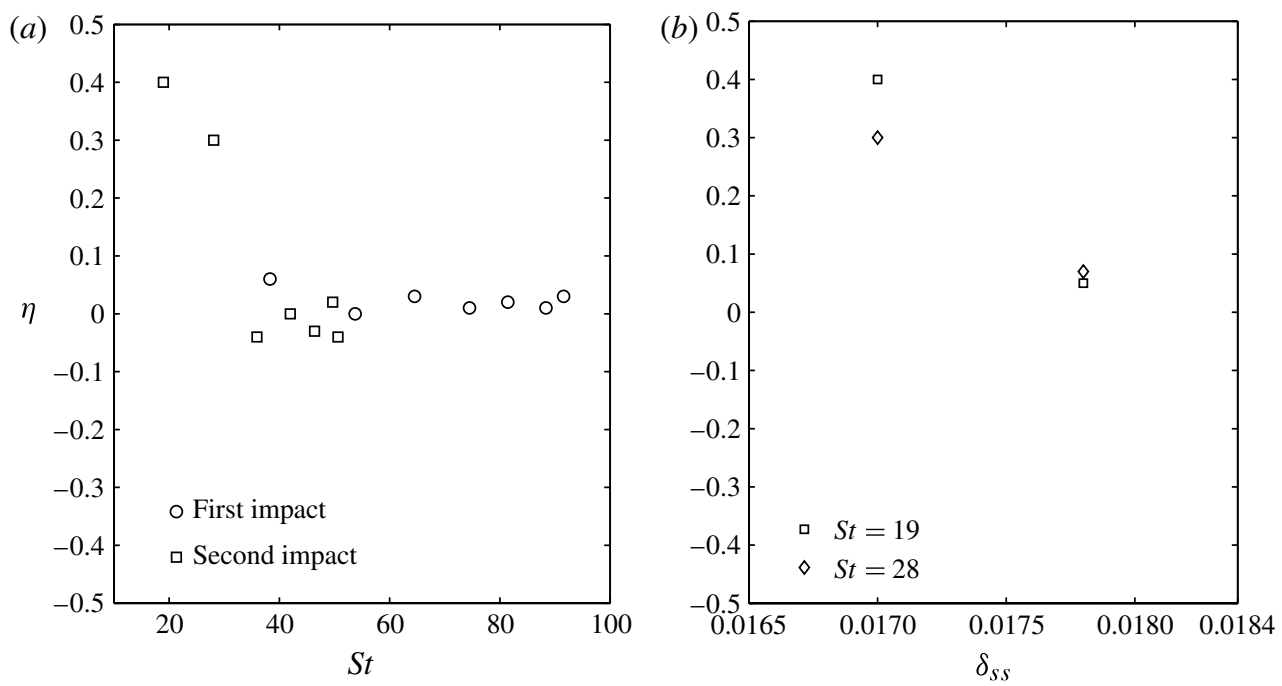

FIGURE 12. Relative error $\eta$ based on maximum rebound height. (a) Relative error as a function of Stokes number. The results of the first and second impacts for the seven cases are presented using $\delta_{s s}=0.017$ for the simulations. ( $b$ ) Relative error as a function of $\delta_{s s}$.

$\eta=\left(H_{s}-H_{e}\right) / H_{e}$ is used where $H_{s}$ and $H_{e}$ are the maximum height that the particle reached during rebound in the simulation and in the experiment, respectively.

Figure 12 shows the relative error for the first and second impacts for each case. The relative error is plotted as a function of Stokes number in figure 12(a). For the cases with Stokes numbers ranging from 30 to 100, the relative error is small and within the uncertainty of the experiments. For smaller $S t$, such as $S t=28$, the simulation with $\delta_{s s}=0.017$ results in a higher rebound than found in the experiment. The inaccuracy at low $S t$ is also seen in the third rebound in figure 9. To simulate the measured trajectory for lower Stokes number, larger values of $\delta_{s s}$ were tried in different simulations since, as discussed in the previous section, a greater $\delta_{s s}$ results in a lower maximum height achieved in a rebound. The results are shown in figure $12(b)$, which confirms that the relative error decreases when a larger $\delta_{s s}$ is used. With a $5 \%$ increase in $\delta_{s s}$, the relative error falls within the uncertainty of the experimental region. The variation in the value of $\delta_{s s}$ with Stokes number is discussed in $\S 5.5$.

\subsection{Coefficient of restitution}

To examine the current contact model over a larger range of impact Stokes numbers and to compare with more experimental results found in the literature, simulations were run for denser particles and glycerol-water mixtures with different viscosity. The input parameters for each of the simulations are given in table 3. For the runs with the same material properties, the initial distance between the sphere and the wall was varied so that the impact Reynolds number and Stokes number are different. In runs 15, 16 and 17, a sphere with an artificially large density was used to observe larger values of Stokes number (up to $\sim 1000$ ) while keeping the Reynolds number below 250 to ensure that the flow field remains axisymmetric. For runs 1 to $29, \delta_{s s}=0.017$ was used in the contact model. As a comparison, in runs 30 to 35 that have the same material properties as in runs 18 to $24, \delta_{s s}=0.017+5 \%$ is used in the contact model, 


\begin{tabular}{|c|c|c|c|c|c|c|}
\hline Run & $\rho_{l}\left(\mathrm{~g} \mathrm{~cm}^{-3}\right)$ & $\mu(\mathrm{cP})$ & $\rho_{p}\left(\mathrm{~g} \mathrm{~cm}^{-3}\right)$ & $E(\mathrm{GPa})$ & $v$ & Comment \\
\hline $1-3$ & 1.17 & 15.2 & 7.6 & 200 & 0.33 & Steel sphere, $65 \%$ glycerol \\
\hline $4-10$ & 1.20 & 50.0 & 7.6 & 200 & 0.33 & Steel sphere, $78 \%$ glycerol \\
\hline $11-14$ & 1.20 & 50.0 & 11.35 & 16 & 0.44 & Lead sphere, $78 \%$ glycerol \\
\hline 15 & 1.20 & 50.0 & 24.0 & 200 & 0.33 & $\begin{array}{c}\text { Artificial sphere, } 78 \% \\
\text { glycerol }\end{array}$ \\
\hline 16 & 1.20 & 50.0 & 36.0 & 200 & 0.33 & $\begin{array}{c}\text { Artificial sphere, } 78 \% \\
\text { glycerol }\end{array}$ \\
\hline 17 & 1.20 & 50.0 & 48.0 & 200 & 0.33 & $\begin{array}{c}\text { Artificial sphere, } 78 \% \\
\text { glycerol }\end{array}$ \\
\hline $18-2$ & 1.22 & 109 & 7.6 & 200 & 0.33 & Steel sphere, $85 \%$ glycerol \\
\hline $25-29$ & 1.25 & 523 & 7.6 & 200 & 0.33 & Steel sphere, $95 \%$ glycerol \\
\hline $30-35$ & 1.22 & 109 & 7.6 & 200 & 0.33 & Steel sphere, $85 \%$ glycerol \\
\hline
\end{tabular}

TABLE 3. Simulations with different input parameters and the corresponding material description. Runs with the same material properties have different initial distance; thus, the impact Stokes numbers are different. For the viscosity unit, $1 \mathrm{cP}=1 \times 10^{-3} \mathrm{~Pa}$.

which results in different coefficients of restitution for impact Stokes number ranging from 8 to 100 .

The coefficient of restitution is plotted as a function of Stokes number in figure 13. For runs 15, 16 and 17 with large Stokes number $\left(400-10^{3}\right)$, the coefficient of restitution approaches the dry value indicating that the rebound is controlled by the elastic force and not by the fluid forces. When the Stokes number is in the range of 10-200, the hydrodynamic forces exert a greater effect on the particle and the coefficient of restitution decreases as the Stokes number decreases. For runs 25-29, a higher liquid viscosity was used so that the kinetic energy of the sphere is dissipated by viscous effects from the liquid-solid interaction term. The impact velocity is small and the corresponding impact Stokes number is smaller than 10. There is no rebound and the coefficient of restitution is zero. Thus, the hydrodynamic force and the elasticlike force lead to a complete contact model for impact Stokes numbers from 1 to 1000 .

The relation between the coefficient of restitution calculated from the current simulations and the particle-impact Stokes number agrees with the empirical trend, as shown in figure 14 that compares the simulated results with the measured results from the current experiments and Joseph et al's' (2001) pendulum experiments for a steel sphere and Zerodur wall. For Stokes number ranging from 90 to 200, the values of the coefficient of restitution calculated from the current simulations overlap with the experimental results of Joseph et al. (2001). For Stokes number ranging from 30 to 90 where Joseph et al. (2001) has fewer experimental points, the simulated results overlap with the measured results from the current experiments. For Stokes number ranging from 10 to 30 , the coefficient of restitution increases sharply with increasing $S t$ and the experimental data are scattered. Within this lower range of Stokes number, the simulation results are sensitive to the non-dimensional parameter $\delta_{s s}$. When using $\delta_{s s}=0.017+5 \%$ the simulations predict a coefficient of restitution that follows the trend represented by the curve fit by Ruiz-Angulo \& Hunt (2010) of the experimental data by Joseph et al. (2001) and Gondret et al. (2002).

In summary, the different material properties, including the particle elasticity, the liquid viscosity and the densities, are incorporated appropriately in the proposed contact model. The current simulations represent the dependence of the coefficient 


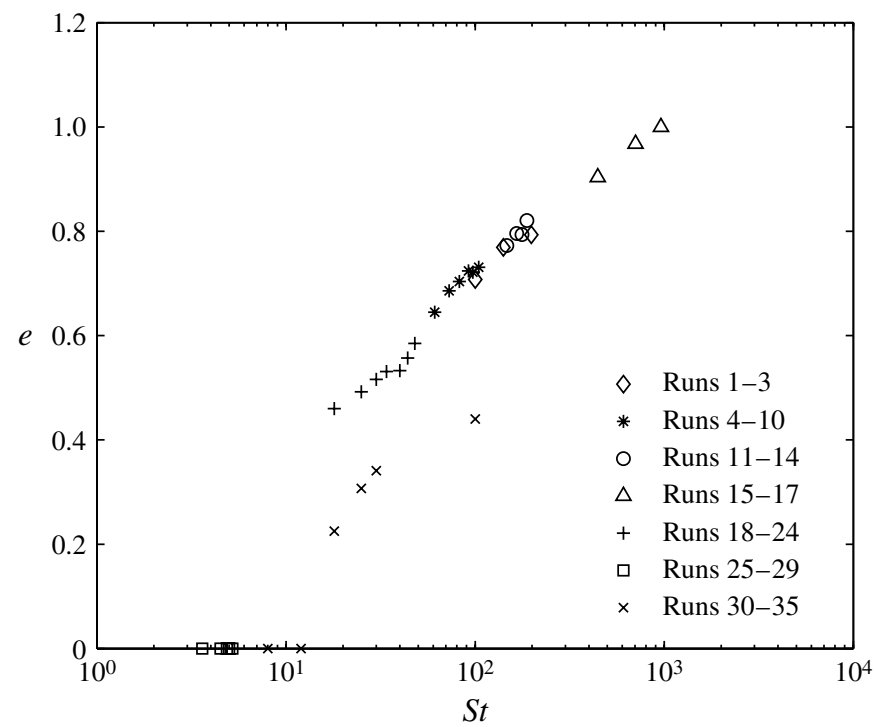

FIGURE 13. Simulation results of coefficient of restitution as a function of Stokes number.

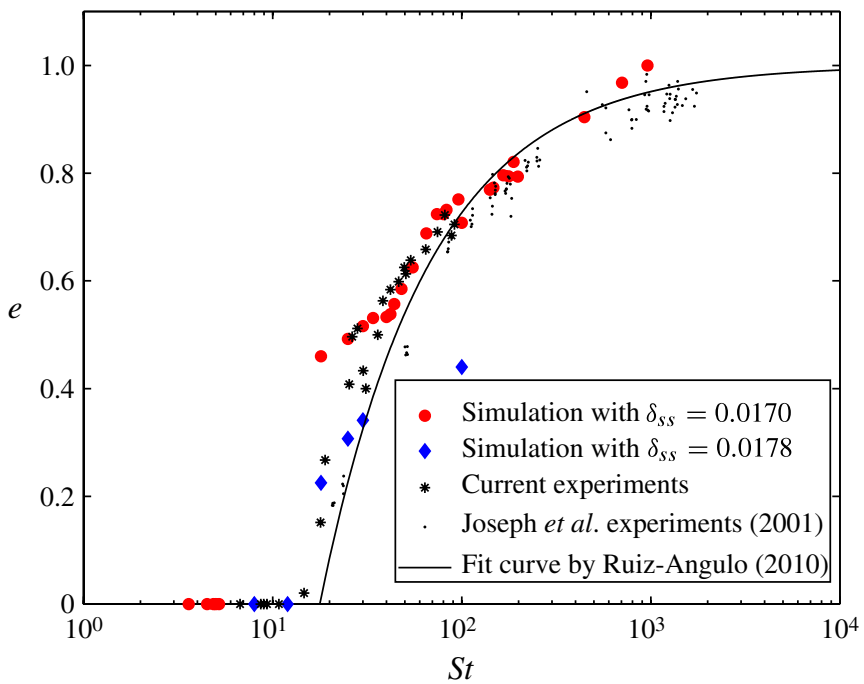

FIGURE 14. (Colour online available at journals.cambridge.org/flm) Comparison of the relations between coefficient of restitution and particle Stokes number.

of restitution on the impact particle Stokes number, demonstrating that the contact model captures the essential physics of a particle-wall collision process in a liquid environment.

\subsection{Discussion of the parameter $\delta_{s s}$}

In the current simulations, the calculated results are influenced by the value of the non-dimensional parameter $\delta_{s s}$ taken in the contact model. At the same Stokes number, a larger $\delta_{s s}$ leads to a lower coefficient of restitution. For Stokes number larger than 30, 
the simulations with $\delta_{s s}=0.017$ produce the good agreement with experimental results for the trajectory and the coefficient of restitution. For Stokes number less than 30 but larger than 10 , the simulation predicts a higher coefficient of restitution when using $\delta_{s s}=0.017$; a lower coefficient of restitution that agrees with the experimental result is obtained when using $\delta_{s s}=0.017+5 \%$. The combined effect of the liquid-solid interaction and the elastic force terms is believed to be the reason. From (4.7) and (4.8), the elastic-like force depends on the impact velocity at $\delta_{s s}$. In simulations with Stokes number less than 30, when $\delta_{s s}<\delta<\delta_{s l}$, the particle velocity decreases markedly because of the effect of the liquid-solid interaction term, as discussed in $\S 5.1$. Thus, a smaller impact velocity results in a smaller elastic force that leads to lower dissipation and a higher rebound velocity. When using $\delta_{s s}=0.017+5 \%$ (runs 30-35), the elastic force term is activated earlier where the particle velocity is larger than the value at $\delta_{s s}=0.017$. As a result, $W_{o}$ is larger and the larger elastic-like term results in large deceleration. The combined effect of the liquid-solid interaction term and the elastic term provides more dissipation for the collision process, which also explains the reason why run 35 using $\delta_{s s}=0.017+5 \%$ predicts lower $e$ at $S t=100$ as shown in figure 14 . For the lower Stokes numbers, increasing $\delta_{s s}$ also has the effect of increasing the effective radius $\left(a+\delta_{s s}\right.$ increases from 0.5170 to 0.5178$)$; for an actual collision under these conditions, the particle would begin to deform farther from the wall and the minimum distance of approach would be larger. A similar increase in the minimum distance of approach with decreasing Stokes number was found in the elastohydrodynamic studies of Davis et al. (1986). The results for the coefficient of restitution for runs 30-34 fall within the experimental uncertainty.

\section{Conclusions}

This paper presents a contact model to simulate numerically the normal collision between a particle and a wall in a liquid environment. The contact model computes the liquid force on the particle using a theoretical formula when it is too close to the wall for the simulated force to be accurate. Instead of solving the complicated coupling between the fluid dynamics and the elastic solid deformation, the model introduces an elastic force in the equation of motion of the sphere; this elastic force provides an energy storage mechanism that allows the particle to rebound while remaining rigid. By including this contact model, a particle settling and rebounding from a wall is simulated using a fast IB method developed in cylindrical coordinates. To validate and calibrate the contact model, experiments are performed on a steel sphere falling from different heights and rebounding from a planar wall. The numerical simulation captures the trajectory of the sphere with multiple bounces and facilitates the calculation of the vorticity dynamics associated with the particle moving downwards and upwards, which has not been investigated in prior studies. When applied to a larger range of impact Stokes numbers, the coefficient of restitution calculated from the simulation increases as a function of the Stokes number following the results of earlier experiments. Thus, the contact model describes the normal immersed collision between a particle and a wall with different particle impact Stokes number as long as the surrounding flow field remains axisymmetric. Future work will focus on applying the contact model to a particle-particle normal collision process in which the target particle is not stationary and on developing a three-dimensional code to simulate oblique collisions and impacts at higher Reynolds number. 


\section{Acknowledgement}

The work was supported by NSF grant 0730284.

\section{REFERENCES}

Al-Samieh, M. F. \& Rahnejat, H. 2002 Physics of lubricated impact of a sphere on a plate in a narrow continuum to gaps of molecular dimensions. J. Phys. D: Appl. Phys. 35, 2311-2326.

ArdekAni, A. M. \& RANGel, R. H. 2008 Numerical investigation of particle-particle and particle-wall collisions in a viscous fluid. J. Fluid Mech. 576, 437-466.

BARNOCKY, G. \& DAVIS, R. H. 1988 Elastohydrodynamic collision and rebound of spheres: experimental verification. Phys. Fluids 31, 1324-1329.

BARNOCKY, G. \& DAVIS, R. H. 1989 The influence of pressure-dependent density and viscosity on the elastohydrodynamic collision and rebound of two spheres. J. Fluid Mech. 209, 501-519.

COlOniUs, T. \& TAIRA, K. 2008 A fast immersed boundary method using a nullspace approach and multi-domain far-field conditions. Comput. Meth. Appl. Mech. Engng 197, 2131-2146.

Crowe, C., Sommerfeld, M. \& Tsuji, Y. 1998 Multiphase Flows with Droplets and Particles. CRC Press.

Davis, R. H., Rager, D. A. \& Good, B. T. 2002 Elastohydrodynamic rebound of spheres from coated surfaces. J. Fluid Mech. 468, 107-119.

DaVis, R. H., SERAYssol, J. \& Hinch, E. J. 1986 The elastohydrodynamic collision of two spheres. J. Fluid Mech. 163, 479-497.

Feng, Z., Michaelides, E. E. \& MaO, S. 2010 A three-dimensional resolved discrete particle method for studying particle-wall collision in a viscous fluid. Trans. ASME: J. Fluids Engng 132, 091302.

Gondret, P., LAnce, M. \& Petit, L. 2002 Bouncing motion of spherical particles in fluids. Phys. Fluids 14 (2), 643-652.

Johnson, T. A. \& PATEL, V. C. 1999 Flow past a sphere up to a Reynolds number of 300. J. Fluid Mech. 378, 19-70.

Joseph, G. G. \& Hunt, M. L. 2004 Oblique particle-wall collisions in a liquid. J. Fluid Mech. 510, 71-93.

Joseph, G. G., Zenit, R., Hunt, M. L. \& Rosenwinkel, A. M. 2001 Particle-wall collisions in a viscous fluid. J. Fluid Mech. 443, 329-346.

Kim, I., Elghobashi, S. \& Sirignano, W. A. 1998 On the equation for spherical-particle motion: effect of Reynolds and acceleration numbers. J. Fluid Mech. 367, 221-253.

LADD, A. J. C. 1997 Sedimentation of homogeneous suspensions of non-Brownian sphere. Phys. Fluids 9, 491-499.

LAmb, M. P., Dietrich, W. E. \& SKlar, L. S. 2008 A model for fluvial bedrock incision by impacting suspended and bed load sediment. J. Geophys. Res. 113, F03025.

Leweke, T., Thompson, M. C. \& Hourigan, K. 2004 Vortex dynamics associated with the collision of a sphere with a wall. Phys. Fluids 16 (9), L74-L77.

LI, X. 2010 An experimental and numerical study of normal particle collisions in a viscous liquid. $\mathrm{PhD}$ thesis, California Institute of Technology.

Lorenzini, G. \& MazZA, N. 2004 Debris Flow Phenomenology and Rheological Modelling. WIT Press.

Michaelide, E. E. 1997 Reviewcthe transient equation of motion for particles, bubbles, and droplets. Trans. ASME: J. Fluids Engng 119, 223-247.

NGUYEN, N.-Q. \& LADD, A. J. C. 2002 Lubrication corrections for lattice-Boltzmann simulations of particle suspensions. Phys. Rev. E 66 (046708).

Ruiz-Angulo, A. \& Hunt, M. L. 2010 Measurements of the coefficient of restitution for particle collisions with ductile surfaces in a liquid. Granul. Matt. 12, 185-191.

SAFA, M. M. A. \& GohaR, A. 1986 Pressure distribution under a ball impacting a thin lubricant layer. Trans. ASME: J. Tribology 108, 372-376.

TAIRA, K. \& ColoniUs, T. 2007 The immersed boundary method: a projection approach. $J$. Comput. Phys. 225, 2118-2137. 
Tencate, A., Nieuwstad, C. H., Derksen, J. J. \& Van Den Akker, H. E. A. 2002 Particle imaging velocimetry experiments and lattice-Boltzmann simulations on a single sphere settling under gravity. Phys. Fluids 14 (11), 4012-4025.

Thompson, M. C., Leweke, T. \& Hourigan, K. 2007 Sphere-wall collisions: vortex dynamics and stability. J. Fluid Mech. 575, 121-148.

Timoshenko, S. P. \& Goodier, J. N. 1970 Theory of Elasticity, 3rd edn. McGraw Hill.

YANG, F.-L. 2006 Interaction law for a collision between two solid particles in a viscous liquid. $\mathrm{PhD}$ thesis, California Institute of Technology.

YAng, F.-L. \& Hunt, M. L. 2006 Dynamics of particle-particle collisions in a viscous liquid. Phys. Fluids 18 (121506). 